\title{
In vitro analysis of the effect of Flightless I on murine tenocyte cellular functions
}

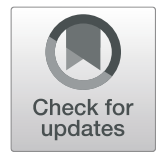

\author{
Jessica E. Jackson ${ }^{1}$, Zlatko Kopecki ${ }^{1}$, Peter J. Anderson ${ }^{2}$ and Allison J. Cowin ${ }^{1 *}$ (D)
}

\begin{abstract}
Background: Healing of tendons after injury involves the proliferation of tenocytes and the production of extracellular matrix; however, their capacity to heal is limited by poor cell density and limited growth factor activity. Flightless I (Flii) has previously been identified as an important regulator of cellular proliferation and migration, and the purpose of this study was to evaluate the effect of differential Flii gene expression on tenocyte function in vitro.

Methods: The role of Flii on tenocyte proliferation, migration, and contraction was assessed using established assays. Tenocytes from Fliit ${ }^{+-}$, wild-type, and Flii overexpressing mice were obtained and the effect of differential Flii expression on migration, proliferation, contraction, and collagen synthesis determined in vitro. Statistical differences were determined using unpaired Student's $t$ test and statistical outliers were identified using the Grubbs' test.

Results: Flii overexpressing tenocytes showed significantly improved migration and proliferation as well as increased collagen I secretion. Explanted tendons from Flii overexpressing mice also showed significantly elevated tenocyte outgrowth compared to Flii ${ }^{+/-}$mice. In contrast to its role in dermal wound repair, Flii positively affects cellular processes in tendons.

Conclusions: These findings suggest that Flii could be a novel target for modulating tenocyte activity and improving tendon repair. This could have significant clinical implications as novel therapeutic targets for improved healing of tendon injuries are urgently needed.
\end{abstract}

Keywords: Flii, Flightless I, Tendon, Healing

\section{Background}

Tendons inherently have minimal capacity for healing owing to poor cell density and growth factor activity [1]. Healing of tendons involves the proliferation of spindleshaped fibroblast-like cells known as tenocytes, which comprise the main cellular content of mature tendons. Tenocytes have long cellular processes which facilitate cell-extracellular matrix (ECM) and cell-cell communication through desmosomes and tight junctions [2]. They are highly metabolically active and are responsible for synthesizing and secreting ECM. Tendon repair is characterized by the influx of intrinsic and extrinsic

\footnotetext{
* Correspondence: allison.cowin@unisa.edu.au

${ }^{1}$ Regenerative Medicine, Future Industries Institute, University of South Australia, Adelaide, South Australia, Australia

Full list of author information is available at the end of the article
}

tenocytes which migrate to the injured area and synthesize and secrete ECM to begin the repair process [3]. Tendon healing can occur solely through epitenonbased intrinsic tenocytes that secrete larger, more mature collagen fibers compared with extrinsic tenocytes, resulting in improved physiological outcomes [4]. Tenocyte characterization during injury repair is poorly understood; however, it is well established that tenocyte function is to secrete ECM components and growth factors during healing [5]. Of particular importance is their secretion of transforming growth factor beta (TGF $\beta$ ) ligands as this family of growth factors is active throughout all phases of tendon repair [5-7].

Tenocyte migration, proliferation, and contraction are essential processes that allow tendon healing to occur. The actin cytoskeleton is the driving force behind these 
processes and it also has essential roles in transmembrane signaling and healing $[8,9]$. In response to injury, the actin cytoskeleton is remodeled to facilitate the permeation of inflammatory cells and promote tenocyte migration $[4,10]$. Flightless (Flii) is a highly conserved actin remodeling protein that contains both a gelsolin and leucine-rich repeat (LRR) domain enabling it to modulate the cytoskeleton by regulating actin polymerization and depolymerization [11-14]. Flii is a known regulator of cellular migration, proliferation, contraction, and adhesion; however, this has been mainly characterized in cutaneous healing responses $[8,15-17]$. Flii is expressed ubiquitously throughout cells and tissues of the body but its specific expression in cells and structures with migratory capabilities is of particular interest due to their involvement with wound repair $[18,19]$. Flii has previously been determined to be an important negative regulator of cutaneous wound healing and skin blistering $[8,14,16-18]$. There is also strong evidence to suggest that Flii modulates TGF $\beta$ expression, with decreased levels of TGF $\beta 1$ detected in cutaneous healing studies in mice with low levels of Flii $\left(\mathrm{Flii}^{+/-}\right)$[20]. In addition, reducing the levels of Flii decreases pro-scarring TGF- $\beta 1$ and Smad 3 , while causing an elevation of anti-scarring inhibitory Smad 7, suggesting a mechanistic role for Flii in TGF- $\beta$ signaling $[16,20]$.

In this study, the effect of altering Flii expression on tenocyte activity was investigated. Primary tenocytes were extracted from digital tendons of $\mathrm{Flii}^{+/}$, wild-type (WT), and Flii ${ }^{T g / T g}$ mice and the effect of differential Flii expression on their migration, proliferation, contraction, and ECM production assessed.

\section{Methods}

\section{Flii mouse generation}

All mouse strains were congenic on the Balb/c background and Balb/c littermates were used as wild-type (WT) control animals. Flii homozygous knockout mice are embryonic lethal due to defects with gastrulation during early embryogenesis [21]. Flii heterozygous mice $\left(\mathrm{Flii}^{+-}\right)$are phenotypically normal, fertile with normal life expectancy. Mice carrying the complete human Flii gene on a cosmid transgene were maintained as described previously $[8,21]$. Heterozygous transgenic mice $\mathrm{Flii}^{\mathrm{Tg} /+}$ were made by crossing $\mathrm{Flii}^{+++}$with cosmid transgene $\mathrm{Flii}^{+/-}$. These transgenic mice were inter-crossed to obtain animals homozygous for the transgene $F l i i^{T g / T g}$ and carry two copies of the mouse Flii gene and two additional copies of the human Flii gene $\left(\right.$ Flii $\left.{ }^{T g / T g}\right)$ resulting in elevated levels of Flii protein in various tissues $[8,19]$.

\section{Isolation of mouse tenocytes}

Murine tenocyte isolation can generally be achieved from adult mice (12-16 weeks) following established protocols [22]. Therefore, digital tendons were removed intact from the hind feet of 4 euthanized female 12-week-old $\mathrm{Flii}^{+/-}$, WT, and Flii ${ }^{T g / T g}$ mice using micro dissection (Fig. 1a). The tendon sheath was removed, and the tip and base of the tendons discarded. The remaining tendons were chopped into $1 \mathrm{~mm}^{3}$ sections and digested overnight in 4 $\mathrm{mg} / \mathrm{mL}$ dispase (Worthington Biochem, NJ, USA), and $0.5 \mathrm{mg} / \mathrm{mL}$ collagenase (Worthington Biochem, NJ, USA) in Dulbecco's modified Eagle's media (DMEM) without serum at $37{ }^{\circ} \mathrm{C}$. The following day, the tendon mix was put through a $70-\mu \mathrm{M}$ cell strainer (In Vitro Technologies, Victoria, Australia) and the remaining tissue discarded. The tube and strainer were washed with sterile media (DMEM $+10 \%$ fetal bovine serum [FBS] + penicillin/ streptomycin $[$ Pen/Strep] + fungizone) and excess media added to stop the action of the dispase/collagenase mix. The suspension was spun at $1200 \mathrm{rpm}$ for $5 \mathrm{~min}$ and the supernatant discarded. The pellet was resuspended in 4 $\mathrm{mL}$ sterile media and plated on collagen-coated T25 flask. The T25 was left in an incubator $\left(37{ }^{\circ} \mathrm{C}, 5 \% \mathrm{CO}_{2}\right)$ for approximately 7 days with media changes every $2-3$ days until the cells were a minimum of $90 \%$ confluent $[23,24]$.

\section{Migration assay}

Tenocytes isolated from $\mathrm{Flii}^{+/-}$, WT, and $\mathrm{Flii}^{\mathrm{Tg} / \mathrm{Tg}}$ mice were plated into 96-well plates at $5 \times 10^{5}$ cells $/ \mathrm{mL}$ and left overnight in an incubator at $37{ }^{\circ} \mathrm{C}$ at $5 \% \mathrm{CO}_{2}$ to reach confluence. A Woundmaker ${ }^{\mathrm{rm}}$ (Essen Bioscience, Michigan, USA) was used to create uniform wounds of $7-800 \mu \mathrm{M}$ in each well of the 96-well plate which was subsequently placed into an Incucyte (Essen Bioscience, Michigan, USA) at $37{ }^{\circ} \mathrm{C}$ and $5 \% \mathrm{CO}_{2}$ where images were automatically taken every $3 \mathrm{~h}$ for $24 \mathrm{~h}$. The resulting images were analyzed using Image Pro Plus 7.1 as previously described [25].

\section{Tendon outgrowth assay}

Whole tendons were removed in sterile conditions from the hind feet of $6 \mathrm{Flii}^{+/-}$, WT, and Flii ${ }^{\mathrm{Tg} / \mathrm{Tg}}$ mice. Five millimeters was removed from the ends of each tendon and the remaining tendon was cut into $3 \mathrm{~mm}^{3}$ sections, transferred into 12 -well plates (1 section per well), and cultured in DMEM + 20\% FBS + Pen/Strep + Fungizone. Cultures were maintained at $37{ }^{\circ} \mathrm{C}$ at $5 \% \mathrm{CO}_{2}$ for 12 days [26]. Images were taken at days $0,4,8$, and 12. Migration distances were measured every $90^{\circ}$ using Image Pro Plus 7.1 (MediaCybernetics Inc., Maryland, USA) and the average distance calculated.

\section{Collagen immunoassay}

A collagen immunoassay was employed similar to previous studies [27]. Briefly isolated $\mathrm{Flii}^{+/-}$, WT, and $\mathrm{Flii}^{\mathrm{Tg} / \mathrm{Tg}}$ tenocytes were seeded into 96-well plates at $5 \times 10^{5}$ cells $/ \mathrm{mL}$ in media (DMEM + 20\% FBS + Pen/Strep + Fungizone) and left overnight at $37{ }^{\circ} \mathrm{C} \quad 5 \% \quad \mathrm{CO}_{2}$. 
Following serum starvation, media were replaced with serum-free media plus Pen/Strep and Fungizone and left for $60 \mathrm{~h}$ to allow collagen accumulation. The cells were subsequently washed 3 times in phosphate-buffered saline (PBS), then fixed and permeabilized in ice-cold methanol for $15 \mathrm{~min}$ at $-20{ }^{\circ} \mathrm{C}$. The cells were aspirated and briefly air-dried before being washed 3 times in PBS. Cells were treated with $0.5 \%$ Tween 20 in PBS for 10 min and blocked in PBS $+3 \%$ normal goat serum for 30 min. The cells were incubated in $50 \mu \mathrm{L}$ per well collagen I (\#600-401-103; Rockland, PA; $2 \mu \mathrm{g} / \mathrm{mL}$ ) in PBS and 3\% normal goat serum for $2 \mathrm{~h}$ at room temperature. The cells were washed and incubated in $50 \mu \mathrm{L}$ per well secondary antibody in PBS for $1 \mathrm{~h}$ in the dark at room temperature. Once the secondary staining was complete, the cells were washed in PBS, stained with 4',6-diamindino-2-phenylindole (DAPI) at 1:5000 for $1 \mathrm{~min}$ and analyzed immediately on an Olympus Epifluorescent microscope as previously described [13].

\section{Collagen contraction assay}

Primary tenocytes and fibroblasts isolated from $\mathrm{Flii}^{+/}$, WT, and Flii ${ }^{T g / T g}$ mice tendons or skin, respectively, were trypsinized in $10 \times$ trypsin/EDTA (Sigma-Aldrich, Sydney, Australia) for $5 \mathrm{~min}$ at $37{ }^{\circ} \mathrm{C}$ and quenched using DMEM + 20\% FBS. The cells were spun for $5 \mathrm{~min}$ at $1200 \mathrm{rpm}$ and resuspended in ice-cold DMEM at $1 \times$ $10^{6}$ cells $/ \mathrm{mL}$. 3D collagen gels were prepared by mixing 8 parts of chilled collagen solution $(2 \mathrm{mg} / \mathrm{mL})$ with 1 part $10 \times$ DMEM containing $10 \%$ FBS as previously described [16]. The $\mathrm{pH}$ was adjusted to 7.4 using $0.1 \mathrm{M}$ $\mathrm{NaOH}$. The tenocytes and fibroblasts were added to the collagen gel mixture at $1 \times 10^{5}$ cells $/ \mathrm{mL}$. Five hundred microliters of this mixture was added per well to a flatbottomed 48-well plate and allowed to set for $120 \mathrm{~min}$ at $37{ }^{\circ} \mathrm{C} 5 \% \mathrm{CO}_{2}$. Following this, the gel was carefully dislodged using a $200-\mu \mathrm{L}$ pipette and the gels floated by the addition of $1 \mathrm{~mL}$ DMEM (+ 20\% FCS, Pen/Strep, and Fungizone). Images were taken at 24,48 , and $72 \mathrm{~h}$ post media addition using Olympus Epifluorescent microscope and the images analyzed for degree of contraction using Image Pro-Plus 7.1 as previously described [16].

\section{Immunocytochemistry}

Immunocytochemistry was undertaken as previously described [13]. Briefly, isolated $\mathrm{Flii}^{+/-}, \mathrm{WT}$, and $\mathrm{Flii}^{\mathrm{Tg} / \mathrm{Tg}}$ tenocyte cells were plated onto glass coverslips and fixed and stained for Flii $(2 \mu \mathrm{g} / \mathrm{mL}$; sc-21716, Santa Cruz Biotechnology, CA), TGB $\beta 1(2 \mu \mathrm{g} / \mathrm{mL}$; sc-52893 Santa Cruz Biotechnology CA), $\beta$-tubulin $(2 \mu \mathrm{g} / \mathrm{mL}$, T4026, SigmaAldrich, NSW), collagen I ( $2 \mu \mathrm{g} / \mathrm{mL}$ \#600-401-103, Rockland, PA), Tenascin-C (2 $\mu \mathrm{g} / \mathrm{mL}, \mathrm{sc}-25328$ Santa Cruz Biotechnology CA), and Scleraxis $(2 \mu \mathrm{g} / \mathrm{mL}$, sc- 87425 Santa Cruz Biotechnology CA). Cells were subsequently stained with phalloidin-FITC (2 $\mu \mathrm{g} / \mathrm{mL}$ \#P5282-0.1G, Sigma-Aldrich, NSW) and nuclear counterstain 4,6-diamidino-2-phenyindole DAPI $(2 \mu \mathrm{g} / \mathrm{mL} \quad$ D9564, SigmaAldrich, NSW). The cells were mounted in fluorescent mounting media (DAKO, CA) and viewed under an Olympus Epifluorescent microscope. Average stainspecific fluorescence was measured using AnalySiS (SoftImaging System GmbH, Munster, Germany).

\section{WST-1 proliferation assay}

Methods were as previously described [25]. Briefly, cells were serum-starved for $6 \mathrm{~h}$ to synchronize the cell cycle. The media was then replaced with DMEM $+20 \%$ FBS and incubated at $37{ }^{\circ} \mathrm{C}, 5 \% \mathrm{CO}_{2}$ for 24,48 , or $72 \mathrm{~h}$. Ten microliters WST-1 was added to each well and the absorbance read on a microplate reader at $450 \mathrm{~nm}$ and $600 \mathrm{~nm}$. Cell proliferation was normalized relative to cells with no serum added as control.

\section{Statistical analysis}

Statistical differences were determined following unblinding of the data using GraphPad software (USA) using the unpaired Student's $t$ test. A $p$ value of less than 0.05 was considered statistically significant. A $p$ value of less than 0.01 was considered highly significant. Statistical outliers were determined using the Grubbs' test with a significance level of 0.05 .

\section{Results}

Tenocytes express Tenascin- $\mathrm{C}$ and Scleraxis

Digital tendons were removed from $\mathrm{Flii}^{+/-}$, WT, and Flii ${ }^{T g} / T g$ mice, the tendon sheath was removed by microscopic dissection, and intrinsic tenocytes were isolated by outgrowth or enzyme digestion methods (Fig. 1). Specific care was taken to remove the ends of the tendons to avoid phenotypically different tenocytes at the myotendinous junction (MTJ) and osteotendinous junction (OTJ). Dermal fibroblasts were also isolated from $\mathrm{Flii}^{+/-}$, WT, and $F l i i^{T g / T g}$ by outgrowth or enzyme digestion methods. Tenocytes are structurally similar to fibroblasts and it is impossible to visually distinguish the two celltypes. Tendon-specific markers Tenascin-C and Scleraxis were used to identify tenocytes (Fig. $1 b-d, h-j$ ). Tenascin-C is a glycoprotein expressed specifically in tenocytes [28]. Scleraxis is uniquely expressed in tissues that form tendons and ligaments [29]. Tenascin- $C$ and Scleraxis were expressed in $\mathrm{Flii}^{+/-}$, WT, and $\mathrm{Flii}^{\mathrm{Tg} / \mathrm{Tg}}$ tenocytes with no expression seen in fibroblasts from the same mice, (Fig. 1b-g). Tenascin-C staining was observed to be peri-nuclear whereas Scleraxis staining was observed in the cytoplasm as well as nuclear regions (Fig. $1 \mathrm{~b}-\mathrm{g}, \mathrm{h}-\mathrm{m}$ ). Tenascin-C was significantly higher in $\mathrm{Flii}^{+/-}$tenocytes when compared to WT and Flii ${ }^{\mathrm{Tg} / \mathrm{Tg}}(p$ $=0.042$ and 0.008 , respectively) but no significant 


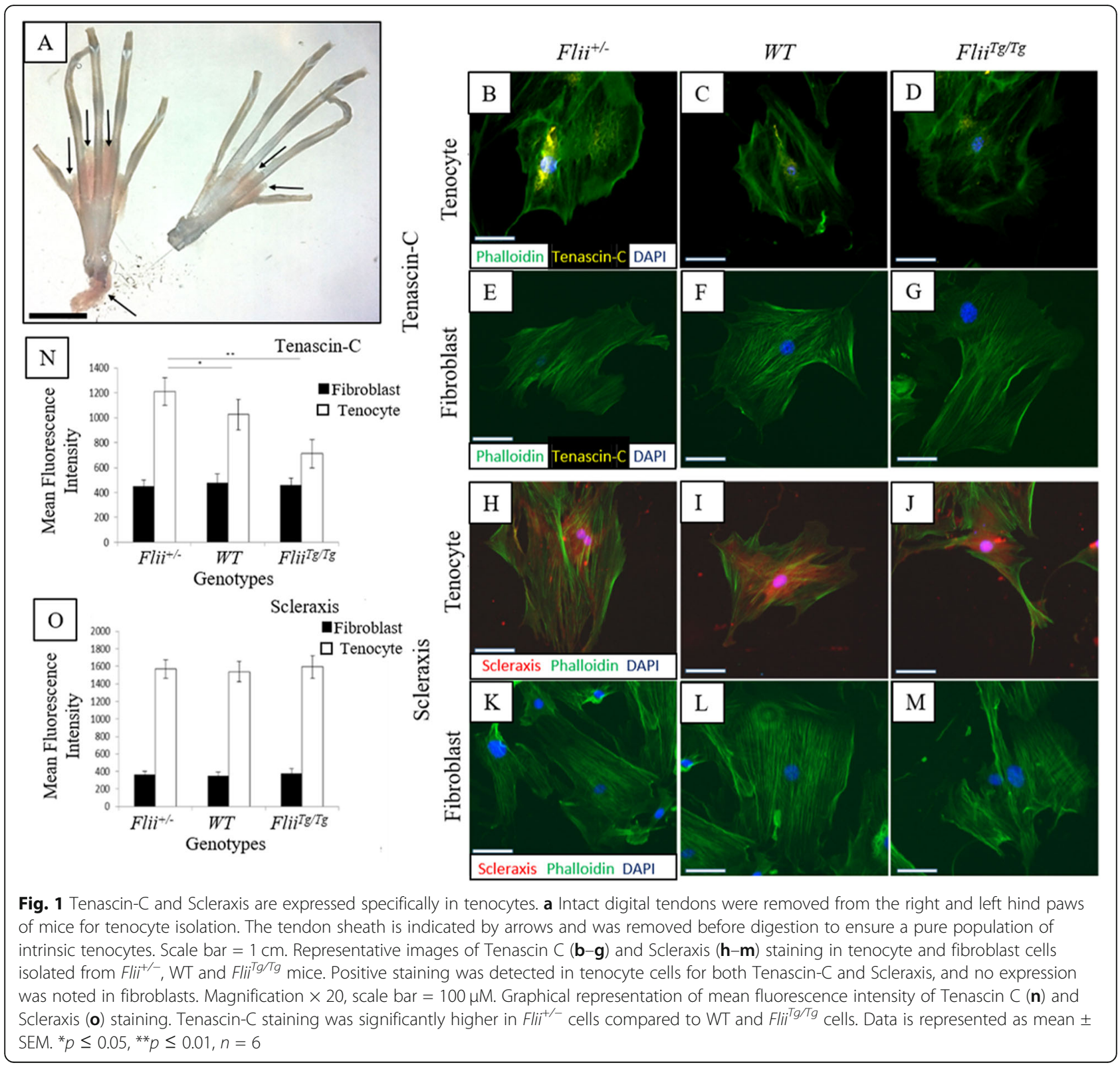

difference was noted in Scleraxis expression in tenocytes across the three Flii genotypes (Fig. 1n, o).

Flii is differentially expressed in $\mathrm{Flii}^{+/-}$, WT, and $\mathrm{Flii}^{\mathrm{Tg} / \mathrm{Tg}}$ tenocytes in response to scratch wounding

Previous studies have shown that Flii is upregulated in fibroblasts in response to wounding [9]. Here we examined Flii levels in wounded $\mathrm{Flii}^{+/-}$, WT, and $\mathrm{Flii}^{\mathrm{Tg} / \mathrm{Tg}}$ tenocytes. Confluent monolayers of tenocytes isolated from $\mathrm{Flii}^{+/-}$, WT, and $\mathrm{Flii}^{\mathrm{Tg} / \mathrm{Tg}}$ mice were scratch wounded and immunofluorescently stained for Flii at 3, 6,12 , and $24 \mathrm{~h}$ post-wounding. Representative images can be seen in Fig. 2a-l. Flii ${ }^{T g / T g}$ tenocytes showed significantly higher Flii expression at 3, 6, 12, and 24 h than
WT $(p=0.0006,0.0002,0.00002$, and 0.00002, respectively) and $\mathrm{Flii}^{+/-}$tenocytes $(p=0.000005,0.00005$, 0.00001 , and 0.00003, respectively) (Fig. 2a-m). Flii staining was apparent at all time points and peaked at $12 \mathrm{~h}$ post-wounding. The staining was mainly perinuclear in distribution, although some cytoplasmic staining was also seen in the later time points.

\section{Tenocyte migration is regulated by Flii}

Previous studies have established the role for Flii in regulating cell migration $[9,15]$. Here we examined the effect of Flii on tenocyte migration and compared it to effects seen in fibroblasts. Isolated primary tenocytes and fibroblasts from $\mathrm{Flii}^{+/-}$, WT, and $\mathrm{Flii}^{\mathrm{Tg} / \mathrm{Tg}}$ mice were 

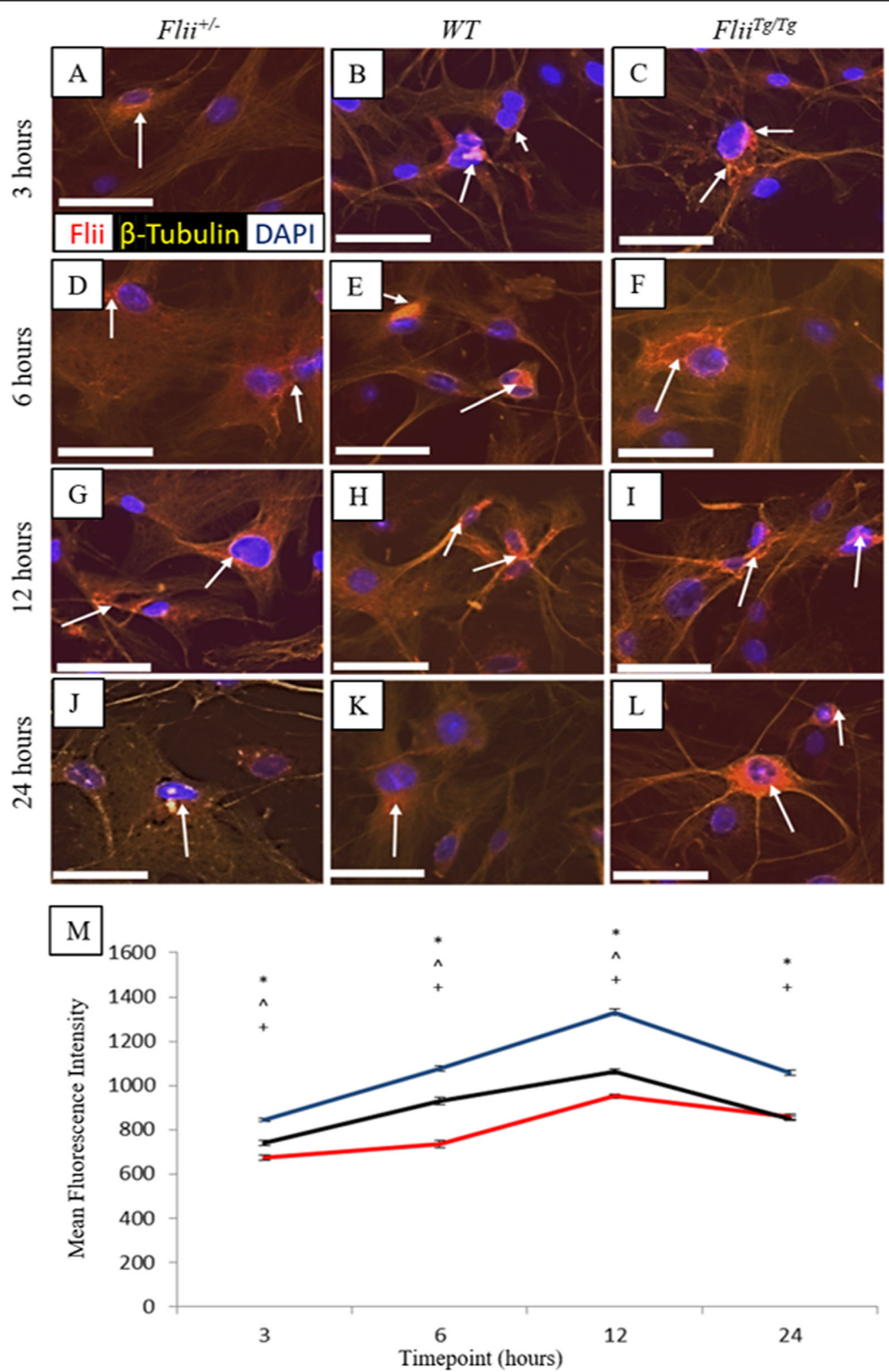

12

-Flii+/-

-WT

Fig. 2 (See legend on next page.) 
(See figure on previous page.)

Fig. 2 Flii expression in Flii ${ }^{+-}$, WT and Flii ${ }^{\text {Tg } / T g}$ tenocytes. Confluent tenocytes were scratch-wounded and stained for Flii expression at 3, 6, 12, and $24 \mathrm{~h}$ post-wounding. a-I Representative images showing Flii expression in Flii ${ }^{+-}, \mathrm{WT}$, and Fli $^{\mathrm{Tg} / \mathrm{Tg}}$ tenocytes at 3, 6, 12 , and $24 \mathrm{~h}$ postwounding. Magnification $\times 10$. Scale bar $=100 \mu \mathrm{M}$. Flii represented by white arrows $(\mathbf{m})$ Graphical representation of Flii expression in tenocytes. $\mathrm{Flii}^{\mathrm{Tg} / \mathrm{Tg}}$ tenocytes express significantly more Flii than WT and Flii ${ }^{+-}$tenocytes. ${ }^{*} p \leq 0.05$ between $\mathrm{Flii}^{+/-}$and $\mathrm{Flii}^{\mathrm{Tg} / \mathrm{Tg}}$. $^{\wedge} \mathrm{p} \leq 0.05$ between $\mathrm{Flii}^{+/-}$and WT. ${ }^{+} p \leq 0.05$ between Flii ${ }^{\text {Tg } T g}$ and WT. Data represented as mean \pm SEM. $n=6$

scratch wounded and cellular migration measured over $24 \mathrm{~h}$. Increased Flii expression resulted in faster cellular migration in tenocytes (Fig. 3a-c) with the Flii ${ }^{T g / T g}$ tenocytes showing significantly faster migration than $\mathrm{Flii}^{+/-}$ tenocytes across the entire time course measured $(p \leq$ 0.001) (Fig. 3g). Flii ${ }^{T g / T g}$ tenocytes also showed significantly faster migration than WT tenocytes at 21 and 24 h post-scratch wounding ( $p=0.05$ and 0.02 , respectively). At $24 \mathrm{~h}$ post-scratch wounding, Flii ${ }^{T g / T g}$ tenocyte wounds had fully closed whereas $\mathrm{Flii}^{+/-}$tenocyte wounds were still $25 \%$ open, suggesting that increased levels of Flii improve the migratory ability of primary murine tenocytes. In contrast, murine fibroblast cells showed that decreased Flii expression improved cellular migration (Fig. 3d-f), with scratch wounded $\mathrm{Flii}^{+/-}$fibroblasts showing significantly faster closure than $F l i i^{T g / T g}$ fibroblasts across the whole time course ( $p=\leq 0.001$ ) (Fig. 3d-f, h).

\section{Tenocyte cellular outgrowth is regulated by Flii}

Flii has previously been shown to regulate cellular outgrowth including both fibroblasts and keratinocytes $[9,15]$. To investigate the effect of Flii on tenocyte cellular outgrowth, $2 \mathrm{~mm}^{3}$ sections of digital tendons, cleaned of their sheaths, from $\mathrm{Flii}^{+/-}$, WT, and $F l i i^{T g / T g}$ mice were placed onto collagen type I coated plates in culture, and the average outgrowth measured over 12 days (Fig. 4a-d). Flii ${ }^{T g / T g}$ tenocytes had significantly increased cellular outgrowth across all 12 days when compared to WT and Flii ${ }^{+/-}$tenocytes ( $p=0.007$ and 0.0005 , respectively, at day 12$)$, with Flii $^{T g / T g}$ cells showing a 2-fold increase in cellular migration by day 12 compared to Flii $^{+/-}$cells (Fig. $4 \mathrm{a}-\mathrm{d}$ ). A dense collection of cells migrated out of Flii ${ }^{T g / T g}$ and WT explants by day 3, whereas $\mathrm{Flii}^{+/-}$showed a less dense, slower initial migration. These cells stained positive for Scleraxis confirming the tenocyte phenotype (data not shown).

\section{Flii ${ }^{T / T g}$ tenocytes have significantly improved proliferation}

Using primary murine tenocytes and fibroblasts from $\mathrm{Flii}^{+/}$, WT, and Flii ${ }^{\mathrm{Tg} / \mathrm{Tg}}$ mice, we examined the effect of altering the level of Flii on proliferation using a 24-h WST-1 assay. In tenocytes, increased Flii resulted in improved cellular proliferation $(p=0.01$ compared to $\mathrm{Flii}^{+/}$) (Fig. 5a). In contrast, fibroblasts showed that increased Flii levels resulted in a decrease in cellular proliferation $\left(p=0.03\right.$ compared to Flii $\left.{ }^{+-}\right)$(Fig. 5b).
Flii has minimal effect on tenocyte contraction $\mathrm{Flii}^{+/-}$, WT, and $\mathrm{Flii}^{\mathrm{Tg} / \mathrm{Tg}}$ tenocytes and fibroblasts were incorporated into a 3D floating collagen gel matrix and left for $72 \mathrm{~h}$ to assess the rate of contraction. Minimal contraction was seen in the tenocyte gels across the $72 \mathrm{~h}$ with no significant difference seen between the genotypes (Fig. 6a-c, g). In contrast, significant contraction was seen in the fibroblast gels across the $72 \mathrm{~h}$, with $\mathrm{Flii}^{+/-}$gels showing significantly increased contraction than WT and Flii ${ }^{T g / T g}$ gels at $48 \mathrm{~h}(p=0.004$ and 0.0001 , respectively $)$ and Flii ${ }^{T g / T g}$ at $72 \mathrm{~h}(p=0.00007)($ Fig. 6d-f, h).

\section{Flii affects collagen type I and collagen type III production in unwounded fibroblasts but not tenocytes} Collagen type I is the main component of tendons, providing stability and strength. Here the effect of Flii on collagen type I and III production in tenocytes and fibroblasts from $\mathrm{Flii}^{+/}$, WT, and $F l i i^{T g / T g}$ mice was investigated. No significant difference was observed in collagen type I production in tenocytes with different levels of Flii; however, fibroblasts were observed to have secreted significantly more collagen type I than the tenocytes (Fig. 6i-o). Overexpression of Flii in Flii ${ }^{T g / T g}$ fibroblasts significantly decreased collagen type I production when compared with fibroblasts from WT and $\mathrm{Flii}^{+/-}$mice $(p=$ 0.0002 and 0.03, respectively) (Fig. 6l-o). Similarly, no significant difference in collagen type III production was observed in the tenocytes across all three genotypes (Fig. 6p-r, v); however, fibroblasts showed an overall significant increase in collagen type III production (Fig. 6s-v) Furthermore, Flii ${ }^{T g} / T_{g}$ fibroblasts showed significantly increased collagen type III secretion when compared to fibroblasts from WT and $\mathrm{Flii}^{+/}$ mice ( $p=0.0002$ and 0.003 , respectively) (Fig. 6v).

\section{Collagen type I production is delayed in scratch-wounded Flii ${ }^{\text {Tg/Tg }}$ tenocytes}

Previous studies have shown that Flii can regulate collagen I production during cutaneous wound healing [8]. Here we examined the effect of differential Flii expression on collagen type I production by wounded tenocytes using established collagen I assay [27]. Tenocytes from $\mathrm{Flii}^{+/-}$, WT, and $F l i i^{\mathrm{Tg} / \mathrm{Tg}}$ mice were scratch-wounded and immunofluorescently stained for collagen type I expression at $3,6,12$, and $24 \mathrm{~h}$ postwounding (Fig. 7a-l). Flii ${ }^{T g / T g}$ tenocytes had a significant delay in peak collagen type I expression at $12 \mathrm{~h}$ post-wounding compared to $\mathrm{Flii}^{+-}$collagen type I expression at $3 \mathrm{~h}$ postwounding; however, the peak level of collagen type I expression was not significantly different (Fig. 7a-m). Collagen type 


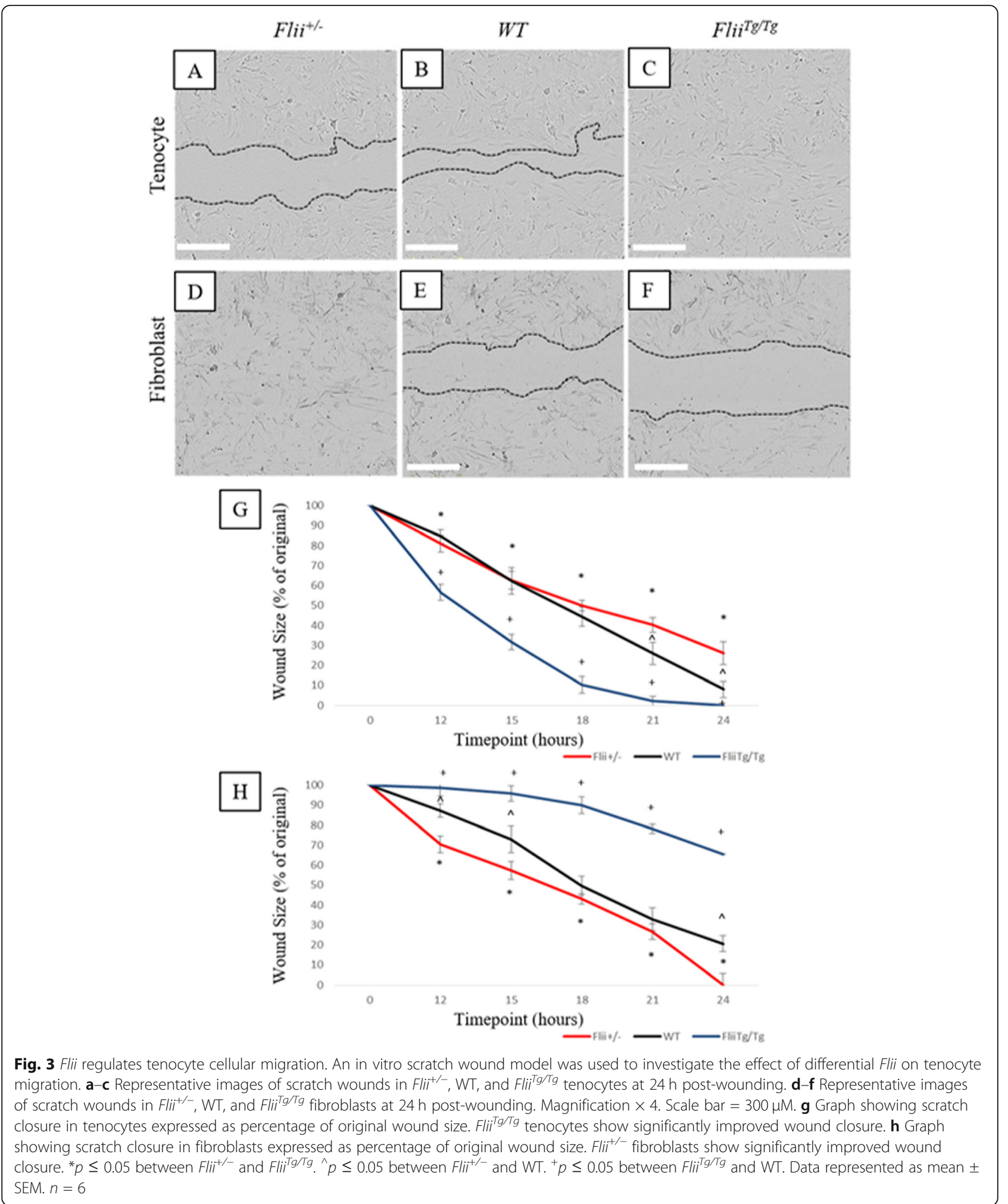

I expression had returned to basal levels in the $\mathrm{Flii}^{+/-}$tenocytes by $12 \mathrm{~h}$ and remained low for the rest of the experiment whereas there was still elevated expression in the $F l i i^{T g / T g}$ tenocytes by $24 \mathrm{~h}$ (Fig. $7 \mathrm{~m})$.
Flii ${ }^{\text {Tg/Tg }}$ tenocytes express significantly less TGF $\beta 1$ during healing

TGF $\beta 1$ is often referred to as the pro-scarring growth factor in the TGF $\beta$ family, and increased levels of this 


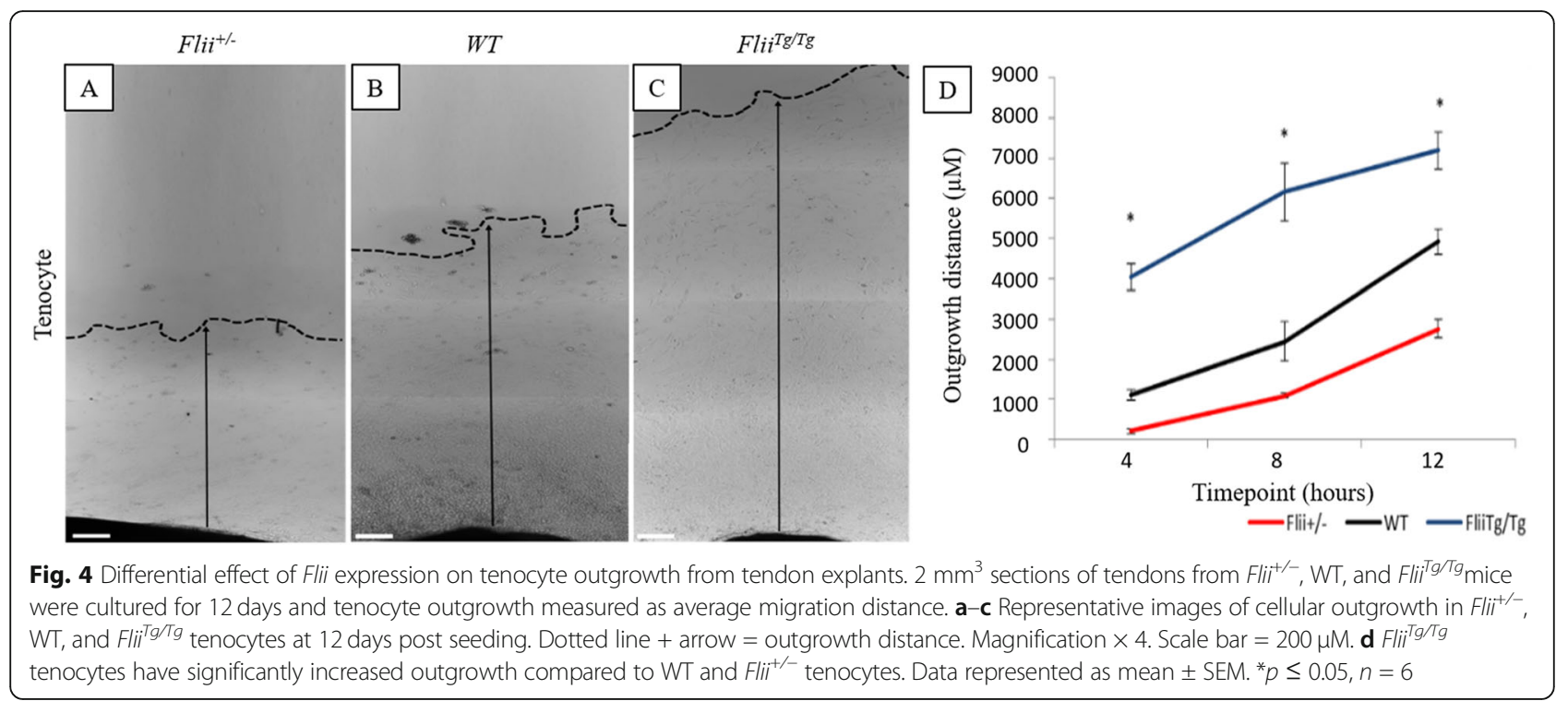

growth factor are generally associated with poorer healing outcomes and more significant scarring and fibrosis [30]. Previous studies have described the effect of differential Flii levels on TGF- $\beta$ expression during wound healing [31]. Here we examined the effect if differential Flii levels on tenocyte TGF- $\beta 1$ expression. Tenocytes from $\mathrm{Flii}^{+/-}$, WT, and $F i i i^{T g / T g}$ mice were scratch-wounded and immunofluorescently stained for TGF $\beta 1$ expression at 3, 6, 12, and $24 \mathrm{~h}$ post-wounding (Fig. 8a-l). TGF $\beta 1$ expression was significantly decreased across all time points in $\mathrm{Flii}^{\mathrm{Tg} / \mathrm{Tg}}$ tenocytes compared to WT $(p=0.03,0.0005,0.003$, and 0.01 , respectively) and $\mathrm{Flii}^{+/-}$tenocytes $(p=0.0003,0.00003$, 0.00007 , and 0.00004 , respectively) (Fig. $8 \mathrm{a}-\mathrm{m}$ ). Tenocytes showed a significant increase in expression between 3-6h, suggesting secretion of TGF $\beta 1$ occurs early in the healing process, with a gradual decline in expression over the remainder of the experiment (Fig. 8m).

\section{Discussion}

Tendon injuries represent a multifaceted model of cellular change, requiring a complex re-organization of the actin cytoskeleton in order to facilitate essential

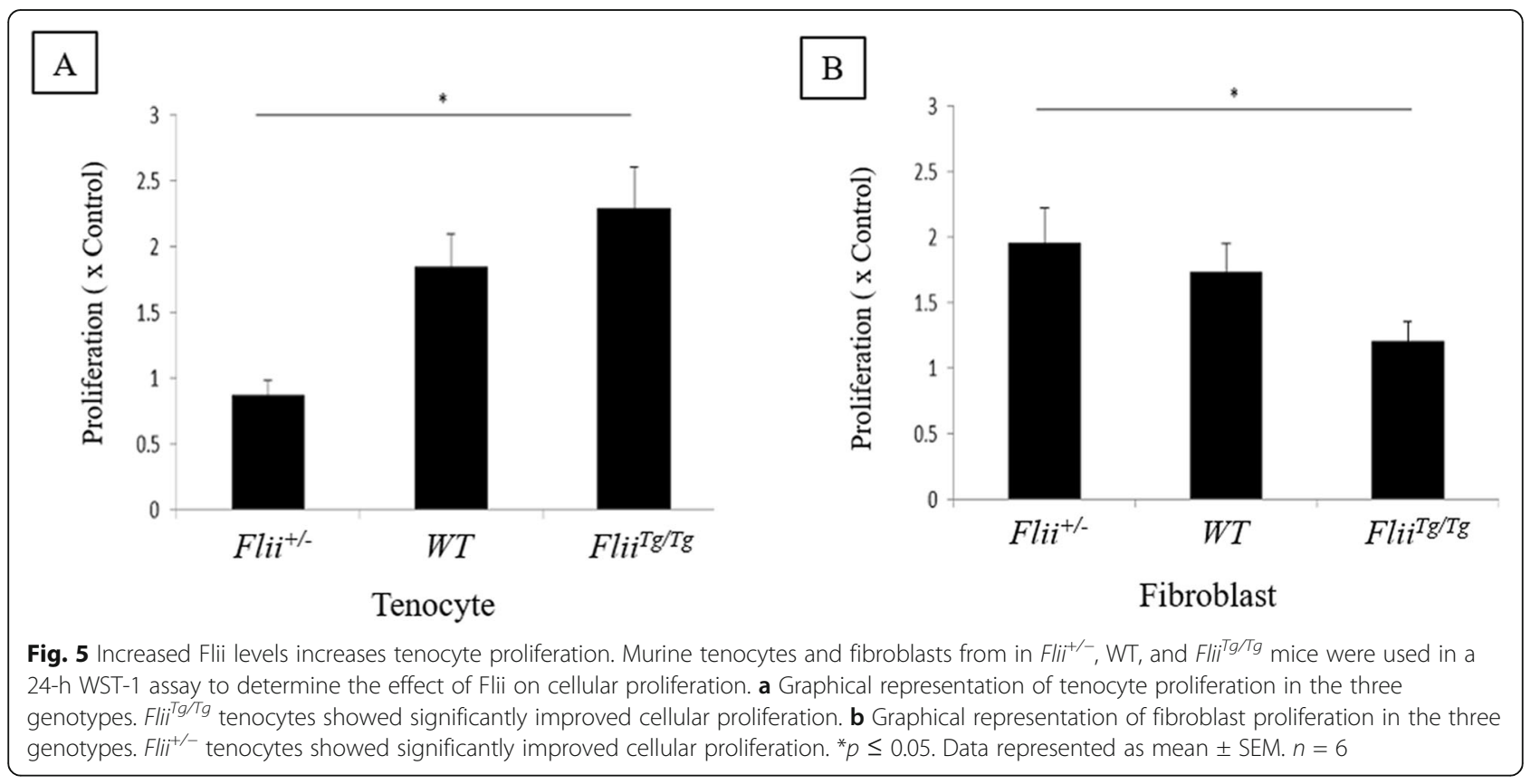




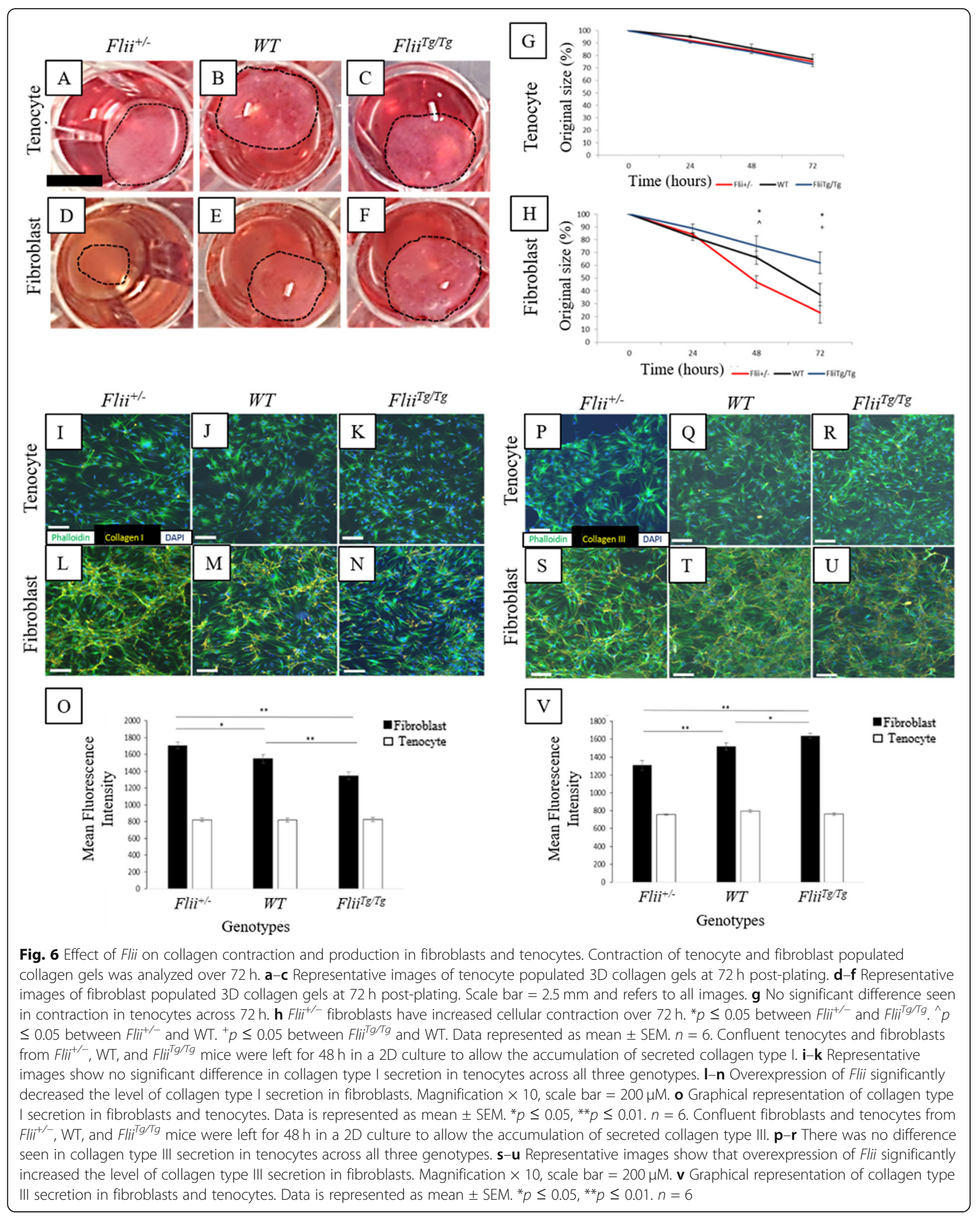



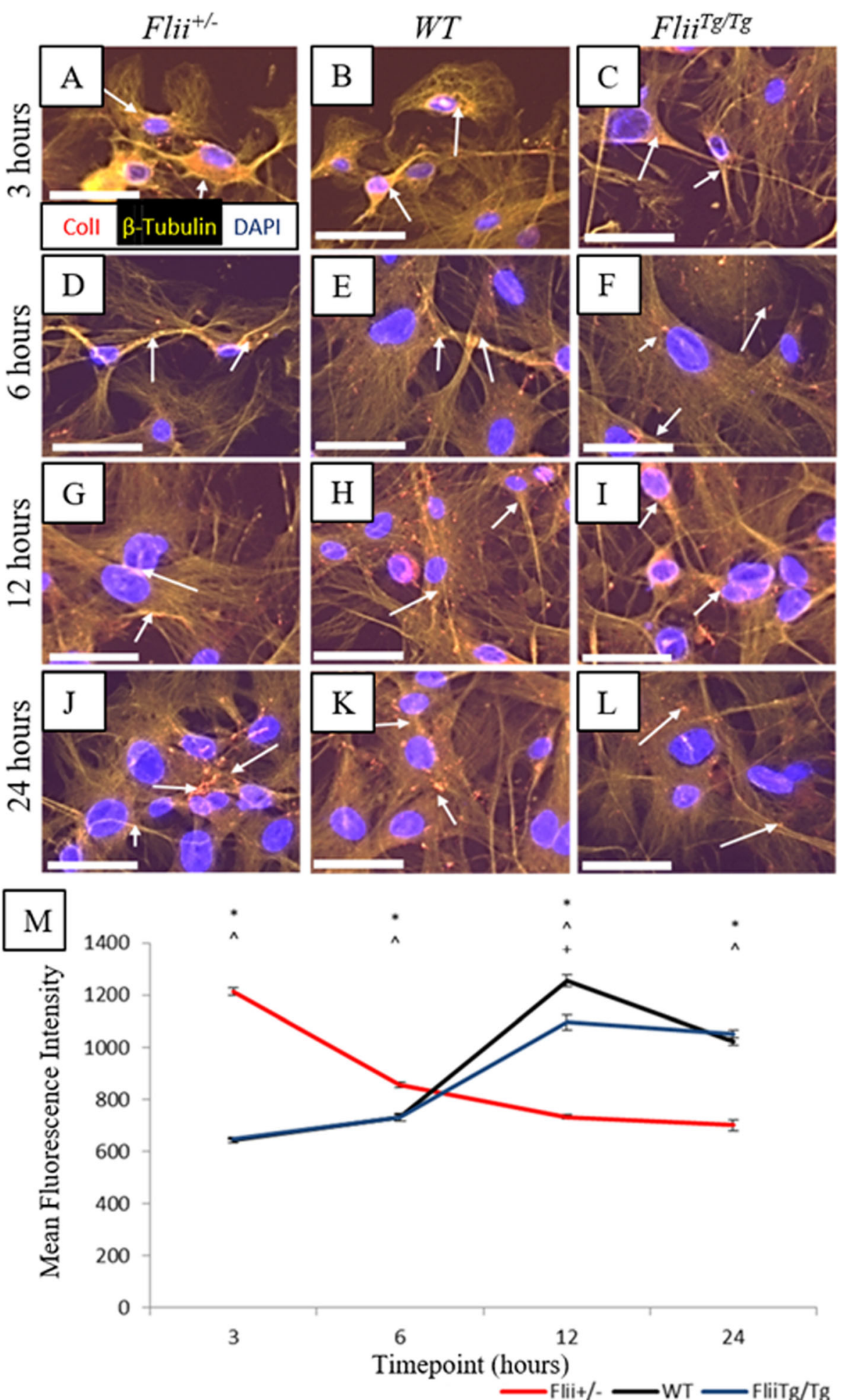

Fig. 7 Flii ${ }^{T / T g}$ tenocytes show decreased collagen type I expression in response to wounding in vitro. Confluent tenocytes were scratch-wounded and stained for collagen type I expression at 3,6, 12, and $24 \mathrm{~h}$ post-wounding. a-I Representative images showing collagen type I expression in $\mathrm{Flit}^{+/-}$, WT, and Flii ${ }^{\mathrm{T} g} / \mathrm{Tg}$ tenocytes at 3, 6, 12, and $24 \mathrm{~h}$ post-wounding. Magnification $\times 10$. Scale bar $=100 \mu \mathrm{M}$. Collagen type I represented by white arrows. $\mathbf{m}$ Graphical representation of collagen type I expression in tenocytes. Flii ${ }^{T / T g}$ tenocytes show a later peak of collagen type I release

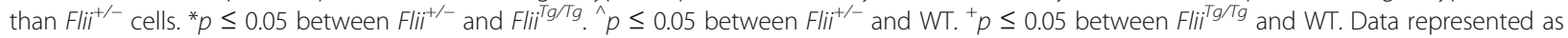
mean \pm SEM. $n=6$ 
processes for healing including migration, proliferation, contraction, and secretion. Tenocytes make up the majority of the cellular content in tendons and are integral in coordinating the healing process following injury [32]. Tenocytes are responsible for releasing signaling molecules to recruit a variety of growth factors, cytokines, and inflammatory cells to the injury site in order to facilitate successful healing [32]. It is well established that both tenocytes and dermal fibroblasts are of mesodermal origin, and both cell types exhibit similar spindle-shaped morphology when grown in in vitro culture. Both produce major extracellular matrix proteins and growth factors in response to injury. For these reasons, tenocyte function is often compared to dermal fibroblasts; however, it is important to note that they are distinct cell types. Tenocytes are mechanosensitive cells with unique properties that detect and respond to changes in mechanical load via deformation of their cell membrane and actin cytoskeleton [33]. Our findings that tenocytes and fibroblasts exhibit different cellular responses to changes in Flii gene expression aligns with previous studies that have shown tenocytes behave differently to dermal fibroblasts in vitro [34] as well as previous studies that show Flii positively affects digit regeneration and rodent vibrissa healing $[35,36]$.

Previous studies have shown that the upregulation of Flii is associated with impaired migration, proliferation, and adhesion of both fibroblasts and keratinocytes $[8,17]$. Flii has previously been reported to act as an intracellular protein that affects actin remodeling and cytoskeleton organization through association with different signaling proteins $[8,11,13]$. Importantly, Flii translocates from the cytoplasm to the nucleus in response to wounding in fibroblasts but not keratinocytes. This suggests that the cell-specific nuclear translocation of Flii may directly regulate gene expression in fibroblasts but not keratinocytes, providing a potential mechanism of action for Flii in wound healing [31]. Using an inducible ROSA26 system to conditionally over-express Flii in fibroblasts resulted in impaired healing responses suggesting that fibroblastspecific production of Flii contributes to wound impaired healing [37]. In this study, increased Flii expression in tenocytes led to an improvement in tenocyte migration, proliferation, and outgrowth, providing more support for the important cell-specific and cell-type-dependent functions of Flii.

Cellular migration is an essential part of the healing process, allowing cells to move across a space created by an injury, to re-establish continuity and strength to an injured area. In order for cells to migrate, a complex cascade of events occurs, with actin assembly driving lamellipodia and filopodia forward, and adhesions forming to anchor the cell body to the surface [9]. Rear adhesions disassemble to allow the cell to retract its rear edge and the cell to move forward. Actin reassembly is integral to this process and actin remodeling proteins such as Flii are essential to ensure this progression occurs correctly [9]. Flii ${ }^{T g / T g}$ tenocytes showed significantly improved migration and proliferation over $24 \mathrm{~h}$ compared with WT and $\mathrm{Flii}^{+/-}$counterparts, indicating the important role of Flii in regulating tenocyte adhesion, migration, and proliferation. These findings are in agreement with previous studies showing Flii association with different signaling proteins involved in actin rearrangement including Cdc42 and the Ras-dependent adenylyl cyclase pathway $[15,38]$. Additionally, studies have shown that Flii regulates focal adhesion turn-over and cell migration by inhibition of paxillin phosphorylation via a Rac-1-dependent pathway $[13,39]$ and its ability to cap actin filaments [40]. Experiments presented in this manuscript show the effects of Flii on tenocyte cell function, further supporting the role of Flii in regulating cellular responses during homeostasis and in response to wounding.

Fibroblasts and tenocytes are both of mesodermal origin and both display similar morphology in vitro as well as being producers of major extracellular proteins such as collagen [41]. Collagen type I is the main component of tendons and tendon adhesions and is an essential protein synthesized during the healing process, providing structure and tensile strength. No significant difference in collagen type I production was observed in the tenocytes while fibroblasts secreted significantly more collagen type I. Overexpression of Flii in Flii ${ }^{T g} /{ }^{T g}$ fibroblasts significantly decreased collagen type I production when compared to fibroblasts from WT and $\mathrm{Flii}^{+/-}$mice. Again, there was no significant difference in collagen type III secretion seen between tenocytes across all three genotypes while fibroblasts showed an overall significant increase in collagen type III production. Flii does have an effect on both collagen type I and collagen type III production as seen in fibroblasts, both in this study and previously [8]. It is, however, the lack of collagen production seen in tenocytes suggest that these cells are less metabolically active than fibroblasts during the "stationary phase" of cell mitosis; this is supported by Stoll et al. that showed slow proliferation and unstable tenocyte phenotype in monolayer cultures [42]. In response to wounding, collagen production in tenocytes is increased and the ratio of collagen type I and collagen type III is also increased [43]. One limitation of this study is the lack of in vivo studies and future in vivo studies in Flii genetic mice will provide more information about the potential effect of differential Flii levels on collagen type I and collagen type III production and organization during the healing of tendon injuries. ECM disorganization is known as a major hallmark in tendinopathy when a significant decrease in type I collagen is prevalent [43]. 

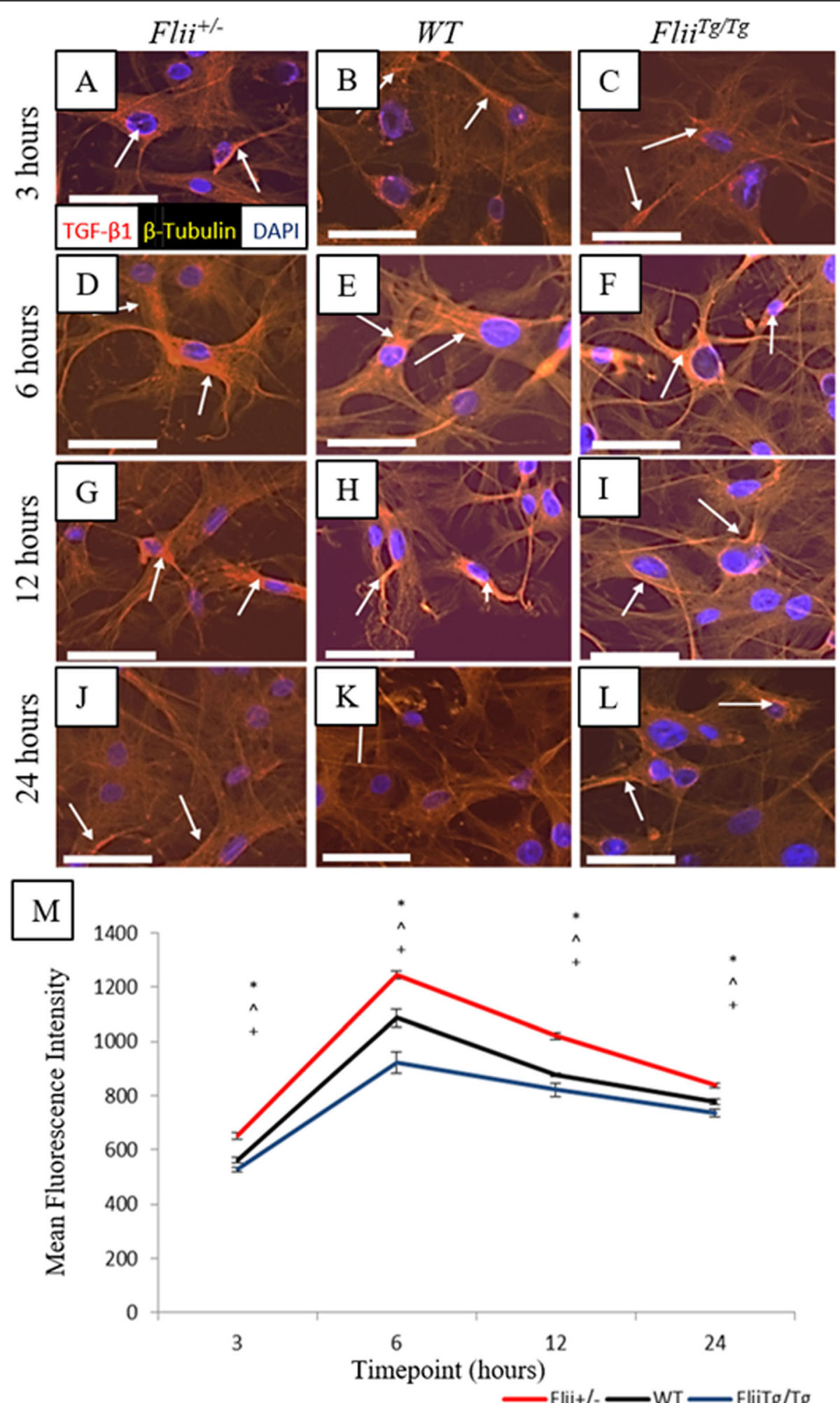

Fig. 8 Flii ${ }^{T / T g}$ tenocytes express significantly less TGF $\beta 1$ than WT or Fliit-- tenocytes following scratch wounding in vitro. Confluent tenocytes were scratch-wounded and stained for TGF $\beta 1$ expression at 3, 6, 12, and $24 \mathrm{~h}$ post-wounding. a-I Representative images showing TGF $\beta 1$ expression in Flit ${ }^{\text {/- }}$ $\mathrm{WT}$, and Flii ${ }^{\mathrm{T} / \mathrm{Tg}}$ tenocytes at 3, 6, 12, and $24 \mathrm{~h}$ post-wounding. Magnification $\times 10$. Scale bar $=100 \mu \mathrm{M}$. TGF $\beta 1$ represented by white arrows. $\mathbf{m}$ Graphical representation of TGF $\beta 1$ expression in tenocytes. TGF $\beta 1$ expression is significantly decreased in Flii ${ }^{\text {TgTg }}$ tenocytes compared to WT and Fliit/- cells. ${ }^{*} p \leq 0.05$ between Flii $^{+-}$and Flii ${ }^{\text {Tg } / T g} .{ }^{\wedge} p \leq 0.05$ between Flii ${ }^{+/-}$and WT. ${ }^{+} p \leq 0.05$ between Flii ${ }^{\text {T/Tg }}$ and WT. Data represented as mean \pm SEM. $n=6$ 
No significant difference was observed between $\mathrm{Flii}^{+/-}$, WT, and Flii ${ }^{T g} / T g$ tenocytes in a 3D collagen gel contraction model suggesting that Flii does not affect tenocyte contraction. Interestingly, this was opposite to previous observations on the effect of Flii on fibroblast collagen gel contraction [16]. Embryonic tenocytes have actinrich fibripositors which act via contraction in order to form the "crimp" structure seen in established tendons. This structure is vital for the mechanical shockabsorbing behavior of mature tendons [44, 45]. Adult tendons show minimal contraction during healing and the "crimp" structure tends to be lost in the reestablished tendon after healing is complete. So, although Flii appears to have minimal effect on adult tenocyte contraction in vitro, Flii is an important developmental regulator $[8,21]$ and has been shown to be involved in fibroblast contraction [16], and so further investigations into its role in embryonic tenocyte cell contraction should be examined.

Many studies have shown that TGF $\beta 1$ is a regulator of fibrosis, and excess TGF $\beta 1$ levels have been associated with poorer healing outcomes and excessive scarring $[46,47]$. This is associated with the inflammatory phase of healing and prolonged expression of TGF $\beta 1$ can lead to an extension of the inflammatory phase which could contribute towards poorer healing outcomes. In a cutaneous burn injury model, elevated Flii leads to increased TGF $\beta$ gene expression and reduced Flii levels are linked with decreased TGF $\beta 1$ expression leading to better healing outcomes [48]. To investigate the possible mechanisms behind which overexpression of Flii in tenocytes may affect collagen deposition, the expression of TGF $\beta 1$ in response to in vitro scratch wounding of tenocytes was investigated. $F l i i^{T g} / T g$ tenocytes expressed significantly less TGF $\beta 1$ compared with their WT and $\mathrm{Flii}^{+/-}$ counterparts. TGF $\beta 1$ has been shown to increase collagen type I production resulting in excessive and disordered organization, leading to increased tendon adhesion size and severity [7]. Flii ${ }^{T g / T g}$ tenocytes expressed significantly lower TGF $\beta 1$ levels during migration, and at $12 \mathrm{~h}$ post-scratch wounding, these cells had significantly lower collagen type I levels than their WT and $\mathrm{Flii}^{+/-}$counterparts. Decreased levels of pro-scarring TGF $\beta 1$ coupled with decreased and more controlled production of type I collagen, by $F l i i^{T g / T g}$ tenocytes, suggest a potential positive role for Flii on healing of tendon injuries.

\section{Conclusions}

Fibroblast behavior has been extensively studied in cutaneous healing experiments, and a reduction in Flii results in improved fibroblast migration, proliferation, adhesion, and contraction $[8,16]$. The opposite is observed in tenocytes in this study suggesting cell-specific effects of Flii may occur in response to different stimuli. This study clearly suggests a positive role for Flii in regulating tenocyte proliferation and migration. In conjunction with decreased collagen type I and TGF- $\beta$ production post wounding, this identifies Flii as a novel target for modulating tenocyte activity and for improving tendon repair. While the exact mechanism of Flii effects on tendon repair is still to be determined, the outcome of this study may help to advance new therapeutic approaches aimed at improving the healing of tendon injuries.

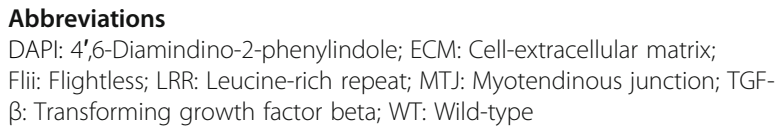

\section{Authors' contributions}

AJC, PJA, and ZK conceived all experiments. JEJ performed all experiments and analysis with the assistance of ZK. All authors contributed to manuscript writing and have approved the final submitted and published versions.

\section{Funding}

This work was supported by an Australian Postgraduate Award scholarship to JEJ. ZK is supported by a Foundation Fellowship from University of South Australia. AJC is supported by NHMRC Senior Research Fellowship GNT\#1102617.

\section{Availability of data and materials \\ The datasets used and/or analyzed during the current study are available from the corresponding author on reasonable request.}

\section{Ethics approval and consent to participate}

All experiments and maintenance of mice were conducted according to Australian Standards for Animal Care under protocols approved by the Women's and Children's Health Network Animal Ethics Committee (WCHN) and carried out in accordance with the Australian code of practice for the care and use of animals for scientific purpose (AE952/9/2016).

\section{Consent for publication}

Not applicable.

\section{Competing interests}

The authors declare they have no competing interests.

\section{Author details}

${ }^{1}$ Regenerative Medicine, Future Industries Institute, University of South Australia, Adelaide, South Australia, Australia. ${ }^{2}$ Faculty of Medicine and Health, University of Adelaide, Adelaide, South Australia, Australia.

Received: 1 March 2020 Accepted: 29 April 2020

Published online: 12 May 2020

\section{References}

1. Legrand A, Kaufman Y, Long C, Fox PM. Molecular biology of flexor tendon healing in relation to reduction of tendon adhesions. J Hand Surg Am. 2017:42(9):722-6.

2. Sharma P, Maffulli N. Tendon injury and tendinopathy: healing and repair. J Bone Joint Surg Am. 2005;87(1):187-202.

3. Wang XT, Liu PY, Tang JB. Tendon healing in vitro: genetic modification of tenocytes with exogenous PDGF gene and promotion of collagen gene expression. J Hand Surg. 2004;29(5):884-90.

4. Sharma P, Maffulli N. Basic biology of tendon injury and healing. Surgeon. 2005;3(5):309-16.

5. Hsu C, Chang J. Clinical implications of growth factors in flexor tendon wound healing. J Hand Surg. 2004;29(4):551-63. 
6. Ngo M, Pham H, Longaker MT, Chang J. Differential expression of transforming growth factor-beta receptors in a rabbit zone II flexor tendon wound healing model. Plast Reconstr Surg. 2001;108(5):1260-7.

7. Klein MB, Yalamanchi N, Pham H, Longaker MT, Chan J. Flexor tendon healing in vitro: effects of TGF- $\beta$ on tendon cell collagen production. J Hand Surg. 2002;27(4):615-20.

8. Cowin AJ, Adams DH, Strudwick XL, Chan H, Hooper JA, Sander GR, et al. Flightless I deficiency enhances wound repair by increasing cell migration and proliferation. J Pathol. 2007;211(5):572-81.

9. Kopecki Z, Cowin AJ. Flightless I: an actin-remodelling protein and an important negative regulator of wound repair. Int J Biochem Cell Biol. 2008; 40(8):1415-9.

10. Molloy T, Wang Y, Murrell GA. The roles of growth factors in tendon and ligament healing. Sports Med. 2003;33(5):381-94.

11. Davy DA, Campbell HD, Fountain S, de Jong D, Crouch MF. The flightless | protein colocalizes with actin- and microtubule-based structures in motile Swiss 3 T3 fibroblasts: evidence for the involvement of PI 3-kinase and Rasrelated small GTPases. J Cell Sci. 2001;114(Pt 3):549-62.

12. Knaup C, Mavroidis P, Stathakis S, Smith M, Swanson G, Papanikolaou N. Evaluation of the effect of prostate volume change on tumor control probability in LDR brachytherapy. J Contemp Brachytherapy. 2011;3(3):12530

13. Kopecki Z, O'Neill GM, Arkell R, Cowin AJ. Regulation of focal adhesions by Flightless I involves inhibition of paxillin phosphorylation via a Rac1dependent pathway. J Invest Dermatol. 2011;131(7):1450-9.

14. Kopecki Z, Yang GN, Arkell RM, Jackson JE, Melville E, Iwata H, et al. Flightless I over-expression impairs skin barrier development, function and recovery following skin blistering. J Pathol. 2014;232(5):541-52.

15. Davy DA, Ball EE, Matthaei KI, Campbell HD, Crouch MF. The flightless I protein localizes to actin-based structures during embryonic development Immunol Cell Biol. 2000;78(4):423-9.

16. Kopecki Z, Arkell RM, Strudwick XL, Hirose M, Ludwig RJ, Kern JS, et al. Overexpression of the Flii gene increases dermal-epidermal blistering in an autoimmune ColVII mouse model of epidermolysis bullosa acquisita. J Pathol. 2011;225(3):401-13.

17. Kopecki Z, Arkell R, Powell BC, Cowin AJ. Flightless I regulates hemidesmosome formation and integrin-mediated cellular adhesion and migration during wound repair. J Invest Dermatol. 2009;129(8):2031-45.

18. Kopecki Z, Ruzehaji N, Turner C, Iwata H, Ludwig RJ, Zillikens D, et al. Topically applied Flightless I neutralizing antibodies improve healing of blistered skin in a murine model of epidermolysis bullosa acquisita. J Invest Dermatol. 2012.

19. Thomsen N, Chappell A, Ali RG, Jones T, Adams DH, Matthaei Kl, et al. Mouse strains for the ubiquitous or conditional overexpression of the Flii gene. Genesis. 2011;49(8):681-8.

20. Chan H, Kopecki Z, Waters J, Powell B, Arkell R, Cowin A. Cytoskeletal protein flightless I differentially affects TGF-beta isoform expression in both in vitro and in vivo wound models. Wound Pract Res. 2014;22(3):169.

21. Campbell HD, Fountain S, McLennan IS, Berven LA, Crouch MF, Davy DA, et al. Fliih, a gelsolin-related cytoskeletal regulator essential for early mammalian embryonic development. Mol Cell Biol. 2002;22(10):3518-26.

22. Shimada A, Wada S, Inoue K, Ideno H, Kamiunten T, Komatsu K, et al. Efficient expansion of mouse primary tenocytes using a novel collagen gel culture method. Histochem Cell Biol. 2014;142(2):205-15.

23. Yao L, Bestwick C, Bestwick L, Maffulli N, Aspden R. Phenotypic drift in human tenocyte culture. Tissue Eng. 2006;12(7):1843-9.

24. Shukunami C, Takimoto A, Oro M, Hiraki Y. Scleraxis positively regulates the expression of tenomodulin, a differentiation marker of tenocytes. Dev Biol. 2006;298(1):234-47.

25. Kopecki Z, Luchetti MM, Adams DH, Strudwick X, Mantamadiotis T, Stoppacciaro A, et al. Collagen loss and impaired wound healing is associated with c-Myb deficiency. J Pathol. 2007;211(3):351-61.

26. Tsai W-C, Hsu C-C, Pang J-HS, Lin M-S, Chen Y-H, Liang F-C. Low-level lase irradiation stimulates tenocyte migration with up-regulation of dynamin II expression. PLoS One. 2012;7(5):e38235.

27. Adams DH, Shou Q, Wohlmuth H, Cowin AJ. Native Australian plant extracts differentially induce collagen I and collagen III in vitro and could be important targets for the development of new wound healing therapies. Fitoterapia. 2016;109:45-51.

28. Taylor SE, Vaughan-Thomas A, Clements DN, Pinchbeck G, Macrory LC, Smith RK, et al. Gene expression markers of tendon fibroblasts in normal and diseased tissue compared to monolayer and three dimensional culture systems. BMC Musculoskelet Disord. 2009;10:27.

29. Murchison ND, Price BA, Conner DA, Keene DR, Olson EN, Tabin CJ, et al. Regulation of tendon differentiation by scleraxis distinguishes forcetransmitting tendons from muscle-anchoring tendons. Development. 2007; 134(14):2697-708

30. Cowin AJ, Holmes TM, Brosnan P, Ferguson MW. Expression of TGF-beta and its receptors in murine fetal and adult dermal wounds. Eur J Dermatol. 2001;11(5):424-31.

31. Chan H, Kopecki Z, Waters J, Powell BC, Arkell R, Cowin AJ. Cytoskeletal protein Flightless I differentially affects TGF- $\beta$ isoform expression in both in vitro and in vivo wound models. Wound Pract Res. 2014;22(3):169-81.

32. Andarawis-Puri N, Flatow EL, Soslowsky L. Tendon basic science: development, repair, regeneration, and healing. J Orthop Res. 2015;33(6): $780-4$

33. Wong JK, Lui YH, Kapacee Z, Kadler KE, Ferguson MW, McGrouther DA. The cellular biology of flexor tendon adhesion formation: an old problem in a new paradigm. Am J Pathol. 2009;175(5):1938-51.

34. Evans $C E$, Trail IA. Fibroblast-like cells from tendons differ from skin fibroblasts in their ability to form three-dimensional structures in vitro. $J$ Hand Surg Br. 1998;23(5):633-41.

35. Strudwick XL, Waters JM, Cowin AJ. Flightless I expression enhances murine claw regeneration following digit amputation. J Invest Dermatol. 2017; 137(1):228-36.

36. Waters JM, Lindo JE, Arkell RM, Cowin AJ. Regeneration of hair follicles is modulated by Flightless I (Flii) in a rodent vibrissa model. J Invest Dermatol. 2011;131:838-47.

37. Turner $C T$, Waters JM, Jackson JE, Arkell RM, Cowin AJ. Fibroblast-specific upregulation of Flightless I impairs wound healing. Exp Dermatol. 2015; 24(9):692-7.

38. Goshima M. Kariya K-i, Yamawaki-Kataoka Y, Okada T, Shibatohge M, Shima F, et al. Characterization of a novel Ras-binding protein Ce-FLI-1 comprising leucine-rich repeats and gelsolin-like domains. Biochem Biophys Res Commun. 1999;257(1):111-6

39. Marei H, Carpy A, Woroniuk A, Vennin C, White G, Timpson P, et al. Differential Rac1 signalling by guanine nucleotide exchange factors implicates FLII in regulating Rac1-driven cell migration. Nature Commun. 2016;7:10664.

40. Mohammad I, Arora PD, Naghibzadeh Y, Wang Y, Li J, Mascarenhas W, et al. Flightless $\mid$ is a focal adhesion-associated actin-capping protein that regulates cell migration. FASEB J. 2012;26(8):3260-72.

41. Tang Q-M, Chen JL, Shen WL, Yin Z, Liu HH, Fang Z, et al. Fetal and adult fibroblasts display intrinsic differences in tendon tissue engineering and regeneration. Sci Reports. 2014:4.

42. Stoll C, John T, Endres M, Rosen C, Kaps C, Kohl B, et al. Extracellular matrix expression of human tenocytes in three-dimensional air-liquid and PLGA cultures compared with tendon tissue: implications for tendon tissue engineering. J Orthop Res. 2010;28(9):1170-7.

43. Thankam FG, Dilisio MF, Gross RM, Agrawal DK. Collagen I: a kingpin for rotator cuff tendon pathology. Am J Transl Res. 2018;10(11):3291-309.

44. Herchenhan A, Kalson NS, Holmes DF, Hill P, Kadler KE, Margetts L. Tenocyte contraction induces crimp formation in tendon-like tissue. Biomech Model Mechanobiol. 2012:11(3-4):449-59.

45. Kapacee Z, Richardson SH, Lu Y, Starborg T, Holmes DF, Baar K, et al. Tension is required for fibripositor formation. Matrix Biol. 2008;27(4):371-5.

46. Crowe MJ, Doetschman T, Greenhalgh DG. Delayed wound healing in immunodeficient TGF- $\beta 1$ knockout mice. J Invest Dermatol. 2000;115(1):3-11.

47. Zhang AY, Pham H, Ho F, Teng K, Longaker MT, Chang J. Inhibition of TGF$\beta$-induced collagen production in rabbit flexor tendons. J Hand Surg. 2004; 29(2):230-5.

48. Adams DH, Ruzehaji N, Strudwick XL, Greenwood J, Campbell H, Arkell R, et al. Attenuation of Flightless $\mathrm{I}$, an actin-remodelling protein, improves burn injury repair via modulation of transforming growth factor (TGF)- $\beta 1$ and TGF- $\beta 3$. Br J Dermatol. 2009;161(2):326-36.

\section{Publisher's Note}

Springer Nature remains neutral with regard to jurisdictional claims in published maps and institutional affiliations. 